\title{
Management of educational and cognitive activity of students within the framework of psychological and pedagogical aspect
}

\author{
Tatyana Micheeva, Elena Murugova* \\ Don State Technical University, Social and Humanitarian Faculty, World Languages and \\ Cultures Department, 344000 Rostov-on-Don, Russia
}

\begin{abstract}
Educational and cognitive activity of students is the key component of the modern educational process. Management of the learning process becomes effective when it is grounded on the basic laws of general theory of management and knowledge of the psychological foundations and cognitive processes patterns, the development and consolidation of certain personal qualities and mental formations that ensure the achievement of educational goals. In this article the main psychological and pedagogical review of management problems is presented; the general requirements for effective learning process management are identified. Particular attention is paid to monitoring the learning process and its correction as a necessary managerial component of the educational and cognitive activity of students.
\end{abstract}

The emergence of the "technologization" idea of education is associated with the search of effective ways of learning process management and, in particular, management of educational and cognitive activity of students, which in its turn would ensure the achievement of guaranteed educational results. Psychological and pedagogical research states that the management of the learning process will be effective and optimal, only when it is built, firstly, on the grounds of the General theory of management (Cybernetics) and, secondly, on the knowledge of the psychological foundations and cognitive process patterns, development and consolidation of certain personal qualities and mental formations ensuring the implementation of educational goals.

In cybernetics, control is perceived as the impact of the control system on the state of the structural elements of the controlled system in accordance with the set goal $[1,2]$. The fundamental principle of a well-managed process is the feedback that regulates the controlled process. This type of good control is called cyclical, in contrast to a poorly managed process without feedback and without regulation of the process. Management in the latter case is called open.

Cyclical management, including the educational process management, should be carried out according to the certain scheme:

1) Identification of the purpose of management;

2) Establishment of the initial state of the managed system;

\footnotetext{
*Corresponding author: murugovaelena@yandex.ru
} 
3) Implementation of the impact program, providing the main transitional states of the system, determined by the specifics of the controlled process, the purpose of control and the initial state of the system;

4) Obtaining information on a specific system of parameters dealing with the state of the managed system at each moment of control (feedback);

5)Pprocessing of information obtained through the feedback channel and the development of corrective (regulatory) effects;

6) Approbation and dissemination of regulatory influences [1, 2, 3].

Psychological and pedagogical management theories differ in the representation way and in the way of completeness of the studied components of the management system, content comprehension and reliance on certain learning theories. The greatest attention is paid to such aspects of management as: target orientation of education, determination of outcomes requirements in the form of standards; teaching approaches of students interaction; organization of cognitive activity of students; organization of control; accounting of results; the use of training tools, etc.

Classification of the educational process management and cognitive activity of students is mainly carried out in compliance with the stated above aspects. In this case, external, behavioral symptoms are mainly called.

Therefore, V.P. Bespalko identifies the type of teaching interaction with the student in the management of cognitive activity according to the following criteria:

1) The presence or absence of control and regulation (cyclic or open control);

2) Orientation (frontal, individual);

3) The use of tools (manual, automated) [2].

In the independent cognitive activity management some researchers (B.P. Esipov, R.M. Mikelson, N.G. Dairy, etc.) usually distinguish the subject of control (authoritarian, democratic, liberal leadership of the teacher or self-management of students) and methods of leadership (activities under the teacher's supervision in accordance with the instructions, samples, algorithms, prescriptions, general rules).

Such a limited approach to the study of managerial procedures in the educational process is characteristic of the traditional (formative) approach of training, when the goals of education are set by a system of subject knowledge and skills. However, even in this case, as the researchers such as N.A. Menchinskaya, V.V. Davydova, P.Y. Halperin and others convincingly showed, the effectiveness of cognitive management is determined not so much by external influences that direct this activity, but by internal ones - the laws of cognitive activity itself. In theories of developing education, this criterion of effective management is considered the leading one.

The most complete problem of cognitive activity of students' management was investigated by N.F. Talyzina, who, based on the general theory of management, revealed the specifics of the stages in the management of the educational process [4].

Management starts by indicating the purpose of the management process. Well managed, technological process requires an instrumental way of setting goals, namely, that the goals should be concrete, defined and recognizable in the measured learning outcomes. Among various ways of determining goals (through the studied content, the internal processes of intellectual, personal development, educational activity), the most efficient is the way of formulating goals through the learning outcomes expressed in observable, recognizable actions. A general technique for such concretization is the use of active verb that indicates a definite action with a specific result (to determine the sequence, build the algorithm, complete the statement, etc.) $[5,6]$. For example, the generalized subject-oriented goal of "mastering the concept of pedagogical technology" in the course of didactics can be concretized with the usage of active verbs:

- the student identifies the essential features of pedagogical technology, 
- the student distinguishes the concepts of "educational technology" and "pedagogical technology",

- the student demonstrates pedagogical technologies used in teaching languages, etc.

As in the learning process not only subject-oriented competences, but also general competences concerning mental and personal activity of students are developed, the educational goal should comprise the following options:

- a system of knowledge and skills with the special indication of mental activity at the level of which the goals should be formed (memory, thinking, etc.);

- system and level of developed indicators (generalization, automation, strength, etc.) (N.F. Talyzina, V.P. Bespalko, etc.)

The next general requirement of effective management is to establish the initial level of students development (knowledge, skills, state of mental activity, personal qualities) without which it is impossible to develop an effective training program.

The central management stage is the development and implementation of the educational process management program that reflects pedagogical impact and its sequence to make students feel involved in cognitive activity. The design of the program focuses on the content and technological part. As the sample of technology realization, we may cover the discipline "Innovative pedagogical technologies in modern professional foreign language education" (master's degree profile in Linguistics "Theory and Methodology of Teaching Foreign Languages and Cultures"). The key used technologies are as follows:

1) Interactive lecture that includes material presentation with the active involvement of students in communication (discussion) to get feedback;

2) Combined visualized lecture, including audio and video materials demonstration;

3) Problem lecture, suggesting a multiple approach to solving problems based on the experience of undergraduates;

4) Seminar on the heuristic plan (solving a problem situation);

5) Project activities;

6) Workshops;

7) Business and role-playing games;

8) Discussions;

9) "Round tables";

10) Training;

11) Questioning and testing.

All listed technologies are grouped as follows:

- cognitive methods (lectures, discussions, round tables, seminars);

- simulation technique (imitates a real situation, solved in interpersonal communication). A number of specific teaching methods is based on simulated technique: role-playing and business games, typical communicative (speech) problems and situations;

- activity exercises (task-solving exercises). They provide an opportunity for active and direct participation of undergraduates in the educational process, the organization of events for the development of communication in the group and educational environment of the faculty, university;

- group-dynamic exercises designed to form and improve communicative competence of undergraduates, culture of business (including professional) communication, the ability to prevent or constructively resolve conflicts, etc. The variety of exercises compared to business games gives reason to consider them as an assessment method and as a training technique. Group-dynamic exercises are built not mainly on the imitation technique but on the free improvisation of students, their spontaneous actions, that can influence the situation and change it; 
- reflective technologies that allow to focus on the state of the participants in training, to work with immediate feelings and sensations, reinforcing positive moods and the effectiveness of the result.

The correlation of the pedagogical impact with the reflection of undergraduates, the management of reflection and mental work in the process of new knowledge assimilation may guarantee a meaningful assimilation of knowledge and the improvement of cognitive culture.

A teacher, based on knowledge of the psychological and pedagogical foundations of modeling the educational process, managing it at all stages of professional activity, does not need inefficient, external, powerful, authoritarian methods of management. He will be guided by:

1) Motivational management through interest, personal significance, problem solving, success situations, etc.

2) Familiarization with the patterns, models and methods of cognitive activity through demonstration of samples, solution of cognitive tasks, special setting of tasks, algorithms and instructions, reflection, etc.

3) Fitting reinforcement of cognitive activity efficiency and unobtrusive analysis of shortcomings.

The process of managing students' knowledge comprehension deserves special attention. The task setting and problem organization of thinking puts before the teacher the necessity to manage students' thinking methodologically competently, provide critical thinking, its subjectification.

To activate cognitive educational activity of students the teacher should go on to manage the self-determination of students, using for this purpose the theory of activity and, in particular, the theory of educational and pedagogical activity.

The next important moment of management is the learning process control and its correction. A teacher should systematically receive information from each student not only about the efficiency of the result, but firstly - whether the performed activity is appropriate and correct. It should be demonstrated with those required characteristics relevant for the formation at this stage.

From a cybernetic point of view, such control, as a feedback, should be prompt and continuous. From a psychological point of view, it should not be excessive; otherwise, it can lead to irritation and loss of interest. In this regard, self-control is more effective, and then only in those cases when the student needs reinforcements.

Moreover, to establish the conformity of the results to the planned goals, the control should rely on clearly fixed criteria of training efficiency. Without such criteria, control is subjective in nature and does not fulfill a managerial function, and does not make it possible to draw up and implement an adequate correctional program. For example, a student does not complete the task, because his cognitive thinking is poor. In addition, the teacher gives him training tasks of the same type, which he still cannot perform. As a result, instead of correction, those mental states that accompanied the student's ineffective activity are fixed insecurity, fear of mistakes and fear of censure, etc.

Thus, the central management stage is the design and implementation of the educational process management program, which reflects pedagogical technologies and its sequence needed for the efficient students' involvement in cognitive activity. At the same time, students should experience cognitive changes in the fixed goals, which are impossible without resorting to specific laws of human cognitive activity, in particular, the course and laws of the knowledge and skills formation, the development of thinking, the formation of personal qualities and the personality as a whole. 
The less rigid the management of the student's cognitive activity, the more it directs the student to problem-solving search, creative tasks, the more it stimulates mental activity and, therefore, is more developing.

\section{References}

1. S. Beer, Cybernetics and management (URSS, Moscow, 2006)

2. V.P. Bespalko, Nature-friendly pedagogy (Narodnoye Obrazovanie, Moscow, 2008)

3. N. Wiener, Cybernetics and society (AST, Moscow, 2019)

4. N.F. Talyzina, Workshop on pedagogical psychology (Academy, Moscow, 2002)

5. M.V. Clarin, Innovation in learning: analysis of foreign experience (Luch, Moscow, 2018)

6. V.N. Andreev, Zh. Fomicheva, N. Kotova, N. Kryukova, E. Murugova and E. Zhukova, Reference Points for the Design and Delivery of Degree Programmes in teaching languages (Deusto, Bilbao, 2013) 